\title{
Cross infection of cystic fibrosis patients with Pseudomonas aeruginosa
}

\section{T L Pitt}

\section{Cross infection with Pseudomonas aeruginosa between} patients with CF has been reported. If this problem becomes widespread, there may be a case for genotyping all strains of $P$ aeruginosa from $\mathrm{CF}$ clinics on a regular basis.

t

t was once stated that pseudomonads are probably the most abundant and widespread life form on the planet. While this may be debatable, there is no doubt that Pseudomonas aeruginosa is one of the most ubiquitous of bacterial species and comprises an almost limitless number of strain populations. Excluding patients with cystic fibrosis (CF), $P$ aeruginosa infections - although associated with high mortality in immunocompromised individuals - are generally manageable. The choice of antimicrobial compounds has not been significantly diminished by resistance, and for most antibiotic groups about $90 \%$ of strains remain susceptible.

It has long been accepted that the lungs of patients with CF become colonised with $P$ aeruginosa from the natural environment and this is reflected by the wide range of strain types recovered from these patients. Indeed, apart from summer camps and group holiday activities where there was good evidence of acquisition of strains from companions with $\mathrm{CF}^{2}$ cross infection between patients attending hospital as inpatients or outpatients was rightly considered to be rare outside CF siblings. In addition to the acquisition of $P$ aeruginosa by previously non-colonised subjects, patients with CF who are already colonised may contract hypertransmissible strains. There were reports of transmission of an antibiotic resistant strain of $P$ aeruginosa in the Copenhagen CF centre in the 1980s, but the methods used then for strain definition lacked discriminatory power and perhaps indicated widespread cross infection when there was none. The technique most widely used today for genotyping bacteria is DNA macrorestriction profiling by pulsed field gel electrophoresis (PFGE) which gives an overview of the architecture of $70-80 \%$ of the genome. It is widely recognised as the gold standard for strain definition because of its superior discrimination and reproducibility over other methods so far evaluated for $P$ aeruginosa. ${ }^{4}$

The first report from Liverpool in 1996 of a ceftazidime resistant strain of $P$ aeruginosa defined by PFGE in children with CF served to raise awareness of a potential problem. ${ }^{5}$ Other communications followed which documented clusters of single strains in some clinics in Melbourne and Wisconsin and culminated in the reports last year from the Liverpool and Manchester adult CF centres. ${ }^{36}$ This prompted a debate as to whether cross infection is widespread in the CF community and led the CF Trust to fund a nationwide survey to determine the extent of the problem. This survey is still in progress but preliminary results suggest that most patients harbour their own strain, although the degree of clustering of strain genotypes varies with centres. For example, in one centre the proportion of patients with strains also found in another individual compared with the proportion with unique strain genotypes varied from 2:11 to $5: 12$, suggesting that in the latter cross infection with multiple strains was more of a problem than in the first centre. Conservation of PFGE profiles between some centres was evident which may indicate the spread of these strains outside the hospital setting. However, further analysis of the genome using a DNA based technique which samples the chromosome randomly at multiple sites (amplified fragment length polymorphism typing) revealed further heterogeneity in these strain populations.

There is therefore evidence to support the occurrence of episodes of cross infection between patients with $\mathrm{CF}$, albeit restricted in scale in most treatment centres. The factors that determine transmissibility are unknown at present but, given the need for $P$ aeruginosa to attach firmly to mucosal surfaces in order to establish the colonised state, one could speculate that adherence differences-perhaps in their affinity for mutant CFTR-might be significant. ${ }^{7}$ The paper by Jones et al in this issue of Thorax suggests that patients who harbour transmissible strains are more difficult to treat than those with unique strains. ${ }^{8}$ If these findings are reproduced elsewhere, there may be a case for genotyping all strains of $P$ aeruginosa on a regular basis from CF clinics to identify and monitor their distribution in patient populations. This would be a significant undertaking with resource implications. However, the results would also inform the infection control strategy and allow more targeted segregation of patients attending clinics.

Thorax 2002;57:921

\section{Author's affiliation}

T L Pitt, Laboratory of Hospital Infection, Central Public Health Laboratory, 61 Colindale Avenue, London NW9 5HT, UK; tpit@@phls.org.uk

\section{REFERENCES}

1 Henwood CJ, Livermore DM, James D, Warner M, and the Pseudomonas Study Group. Antimicrobial susceptibility of Pseudomonas aeruginosa: results of a UK survey and evaluation of the British Society for Antimicrobial Chemotherapy disc susceptibility test I Antimicrob Chemother 2001;47:789-99.

2 Hunfeld KP, Schmidt C, Krackhardt B, et al. Risk of Pseudomonas aeruginosa cross colonisation in patients with cystic fibrosis within a holiday camp: a

molecular-epidemiological study. Wien Klin Wochenschr 2000; 1 12:329-33.

3 McCallum SJ, Corkhill J, Gallagher M, et al. Superinfection with a transmissible strain of Pseudomonas aeruginosa in adults with cystic fibrosis chronically colonised by $\mathrm{P}$ aeruginosa. Lancet $2001 ; 358: 558-60$.

4 Grundmann H, Schneider C, Hartung D, et al. Discriminatory power of three DNA-based typing techniques for Pseudomonas aeruginosa. J Clin Microbio 1995;33:528-34.

5 Cheng K, Smyth RL, Govan JRW, et al. Spread of $\beta$-lactam resistant Pseudomonas aeruginosa in a cystic fibrosis clinic. Lancet 1996;348:639-42

6 Jones AM, Govan JRW, Doherty CJ, et al. Spread of a multiresistant strain of Pseudomonas aeruginosa in adult cystic fibrosis clinic. Lancet 2001;358:557-8.

7 Pier GB, Grout M, Zaidi TS. Cystic fibrosis transmembrane conductance regulator is an epithelial cell receptor for clearance of Pseudomonas aeruginosa from the lung. Proc Natl Acad Sci USA 1997;94:12088-93.

8 Jones AM, Dodd ME, Doherty CJ, et al. Increased treatment requirements of patients with cystic fibrosis who harbour a highly transmissible strain of Pseudomonas aeruginosa. Thorax 2002;57:924-5. 